\section{Ocular immune privilege}

\begin{abstract}
It has been over 60 years since the phrase immune privilege was used by Sir Peter Medawar to describe the lack of an immune response against allografts placed into the ocular microenvironment. Since then, we have come to understand that the mechanisms of ocular immune privilege include unique anatomical features of a blood barrier and a lack of direct lymphatic drainage.

Also, we know that the ocular microenvironment is rich with immunosuppressive molecules that influence the activity of immune cells. Moreover, the placement of foreign antigen into the ocular microenvironment can induce a systemic form of tolerance to the foreign antigen called anterior chamber-associated immune deviation (ACAID). Many soluble immunomodulators are found in aqueous humour, and are a mixture of growth factors, cytokines, neuropeptides, and soluble receptors. This is a continuously growing list. The mechanisms of ocular immune privilege induce apoptosis, promote the production of anti-inflammatory cytokines, and mediate the activation of antigen-specific regulatory immunity. These mechanisms of immune privilege also attempt to impose themselves upon immunity within the uveitic eye. The adaptation of several anatomical and biochemical mechanisms to establish an immune privileged microenvironment within the eye makes the eye immunologically unique. It is a tissue site where we may learn how immunity is regulated in inflammation and at rest. Success in translating the lessons of ocular immune privilege to other tissues has the potential to drastically change the therapy and clinical outcomes of autoimmune diseases and allograft survival.

Eye (2009) 23, 1885-1889; doi:10.1038/eye.2008.382; published online 9 January 2009
\end{abstract}

Keywords: immune privilege; ACAID; immunomodulation; uveitis; neuropeptides

AW Taylor ${ }^{1,2}$

The first experimental description of ocular immune privilege was made by Medawar ${ }^{1}$ in the 1940s. He described the prolonged survival of skin allografts placed within the ocular anterior chamber of rabbits that were already immunized to reject skin allografts. To explain this phenomenon, he noted that the graft survived as long as new vessels did not grow into the transplanted graft. The rejection process appeared to follow new vessel formation. Because of the physical lack of direct lymphatic drainage and the presence of a blood-ocular barrier, he defined the concept of immunological ignorance. He proposed that it was the lack of antigens leaving the graft through lymphatics to draining lymph nodes to induce immune response along with a block in immune cells to enter the tissue through blood vessels was what protected the allografts. Therefore, the tissue was afforded immune privilege through a passive mechanism of immunological ignorance.

This concept was further promoted by Barker and Billingham ${ }^{2}$ where they created in guinea pigs an isolated skin flap that was cut off from their lymphatic drainage and had a vascular umbilical cord-like connection to the blood circulation. When a skin allograft was placed into the centre of this modified skin tissue, there was a prolonged allograft survival very similar to the placement of allografts into the anterior chamber. The experimentation showed that recipient guinea pig was not sensitized to the alloantigens. Rejections of the allografted skin flaps occurred when the lymphatics were reestablished. This gave further support that one of the mechanism of immune privlege is the lack of direct lymphatic drainage. However, unlike the allografts placed in the anterior chamber of immunized hosts, the placement of allografts onto the skin flaps of presensitized hosts lead to rapid graft rejection. This suggests that there was more than just the lack of lymphatics and immunological ignorance that were protecting the allografts in the immune privileged eye.

The work of Kaplan and Streilein ${ }^{3}$ showed that the immune system was not ignorant of

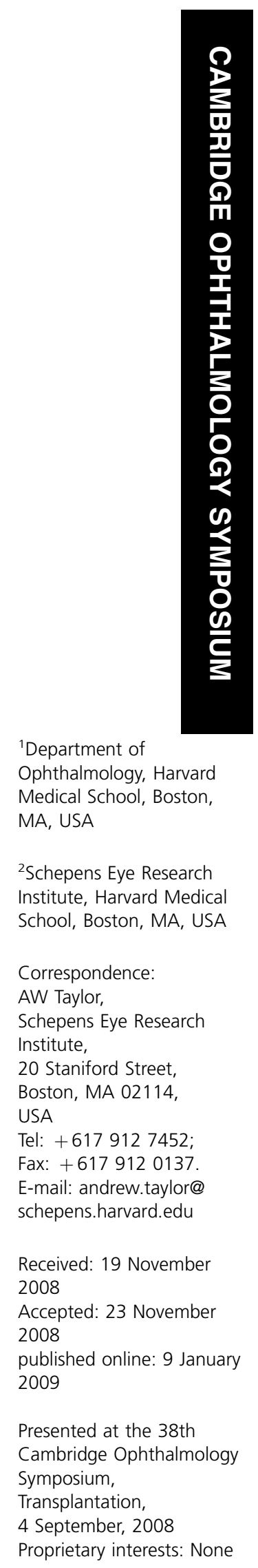


alloantigens placed into the ocular microenvironment, it just made a different immune response. The placement of foreign antigen into the anterior chamber induces suppressor immunity. This suppressor immunity is expressed by the induction of antigen-specific efferent suppressor CD8 T cells and afferent suppressor CD4 T cells, now called $\mathrm{T}_{\text {reg }}$ cells. ${ }^{4}$ Along with the induction of $\mathrm{T}_{\text {reg }}$ cells is the induction of non-complement-fixing antibodies. ${ }^{5}$ As the placement of the same antigens into the skin would induce a potent hypersensitivity response, the process of inducing suppressor immunity by placing the antigen into the anterior chamber is called anterior chamber-associated immune deviation (ACAID). Today we know that the process is mediated by F4/80 macrophages that present the ocular inoculated antigen to a cluster of B cells, NKT cells, CD4, and CD8 T cells in the spleen. ${ }^{6-8}$ The induction of ACAID is tested by sensitizing the host with the antigen 10 days after the ocular inoculation of the antigen followed by a challenge injection of antigen, usually in the ear, 10 days later with the swelling measured over the next $48 \mathrm{~h}$. If ACAID is induced, then the swelling is greatly diminished in comparison to hosts that were sensitized and challenged with the antigen with no ocular inoculation. The induction of ACAID required that the spleen be intact for at least 4 days after inoculating antigen into the anterior chamber and that the eye containing the antigen not be removed before 2 days. ${ }^{9}$ These findings suggested that there is something about the ocular microenvironment that is influencing the presentation of intraocular antigen to induce suppressor immunity. Further experimentation has revealed that an ACAID-like response can be also induced when foreign antigen is placed into the brain. ${ }^{10}$

We now understand that the ocular microenvironment is rich with immunosuppressive molecules that influence the activity of immune cells. Macrophages treated with aqueous humour process antigen and present the antigen in a manner that promotes immune suppression as it is in ACAID. ${ }^{11}$ Aqueous humour inhibits T-cell proliferation in mixed lymphocyte reactions and cytotoxic T-cell activity. ${ }^{12}$ It suppresses IFN- $\gamma$ production and promotes TGF- $\beta$ production by CD4 T cells. ${ }^{13}$ There is a growing list of identified factors in aqueous humour that can influence a range of immune cells or only target specific immune cells. Aqueous humour contains the neuropeptides $\alpha$-MSH, VIP, SOM, the cytokine TGF- $\beta_{2}$, and molecules, such as IDO, and surface expression of FasL to suppress the activation of Th1 cells. ${ }^{14-19}$ The aqueous humour neuropeptides $\alpha$-MSH and CGRP with TGF- $\beta_{2}$ suppress the activation inflammatory activity in macrophages. ${ }^{16,20}$ Recruitment of neutrophils and macrophages maybe regulated by FasL, TGF- $\beta_{2}$, and $\alpha$-MSH. ${ }^{19,21,22}$ The presence of MIF in aqueous humour prevents NK-cell activation, ${ }^{23}$ which is important as there is a lower than normal expression of MHC class molecules. The aqueous humour contains complement factors and their inhibitors. ${ }^{24}$ It may also contain proteins, such as thrombospondin, that contribute to the local activation of TGF- $\beta .^{25}$ Altogether, the healthy ocular microenvironment places a high threshold to be overcome for the induction of inflammation and immunity within its tissue microenvironment.

The composite of immunoregulatory and immunosuppressive activity associated with the molecules produced within the ocular microenvironment suggests that if an efferent immune response occurs within the eye, its inflammatory response should be suppressed with promoted regulatory immunity. The placement of hypersensitivity mediating $\mathrm{T}$ cells into the anterior chamber of the eye along with their antigen, and antigen presenting cells do not mediate inflammation in the eye. ${ }^{26}$ Moreover, if these T cells are recovered from the anterior chamber and adoptively transferred to a conventional tissue site still do not mediate inflammation. The $\mathrm{T}$ cells can be treated in vitro with aqueous humour, and their cytokine profile can be shown to change from IFN- $\gamma$-producing $\mathrm{T}$ cells to TGF- $\beta$-producing T cells. ${ }^{13}$ This can also be replicated by treating the T cells as they are antigen activated with the aqueous humour factors $\alpha-\mathrm{MSH}$ and TGF- $\beta_{2} \cdot{ }^{27}$ These T cells no longer function as hypersensitivity-mediating $\mathrm{T}$ cells, but as regulatory T cells. Pigmented epithelial cells of the iris can also induce regulatory activity in T cells through contact. ${ }^{28}$ Another finding of T cells entering the eye is that many will undergo apoptosis in contact with cells that make up the ocular microenvironment through the expression of FasL. ${ }^{19}$ Recently, it has been found that following an episode of experimental autoimmune uveitis (EAU) there emerges in the spleen retinalantigen-specific $\mathrm{T}_{\text {reg }}$ cells. ${ }^{29}$ These $\mathrm{T}_{\text {reg }}$ cells are not necessary for the normal recovery of the mouse eye in EAU, but have a role in preventing memory immunity to the autoantigens.

The effects of aqueous humour on APC functionality has been described in detail associated with the induction of ACAID. ${ }^{30}$ This has focused on the effects of TGF- $\beta_{2}$ on macrophage APC activity. The aqueous humour factors, such as $\alpha$-MSH and CGRP have an effect on inflammatory activity of macrophages. ${ }^{16,31}$ Not only do they suppress endotoxin-induced inflammatory activity, but induce an anti-inflammatory cytokine production by the macrophages. An $\alpha$-MSH-treated APC cannot activate Th1 cells and the APC suppress IFN- $\gamma$ production by Th1 cells, possibly through IL-10, and $\alpha-\mathrm{MSH}$ production by the treated APC. This suppression of inflammation by innate immunity suggests a possible 
mechanism by which innate immune cells can still clear pathogens and toxins, but not mediate an associated inflammatory response, nor induce an inflammatory adaptive immune response.

The effects of aqueous humour factors on immune cells not only suppress the inflammatory activity, but also induce regulatory activity. ${ }^{13}$ This activity defines immune privilege as also a tissue site of active mechanisms to suppress inflammation and to manipulated immune cells to regulate themselves. Therefore, when there is uveitis, it is interesting to find out what mechanisms are failing. Two publications from the Streilein group in 2000 examined the immunomodulatory activity of the ocular microenvironment in two mouse modules of uveitis, endotoxin-induced uveitis (EIU) and EAU. ${ }^{32,33}$ Both models are a self-limiting ocular inflammation, with EIU lasting $48 \mathrm{~h}$ and limited to the anterior chamber, and EAU can last depending on the strain of mouse from 40 to 60 days and predominately targets the retina. The induction of the two diseases is different with EIU induced by a systemic injection of endotoxin, and EAU induced by immunizing the mice with retinal autoantigen in Freund's adjuvant with a subsequent injection of pertussis toxin.

In EIU, the Streilein group found that the loss of aqueous humour immunosuppressive activity started within the first hour after the injection of LPS. ${ }^{32}$ This preceded by a 4-h detection of elevated protein concentration in aqueous humour, and by $6 \mathrm{~h}$ before there was a detection of proinflammatory cytokines IL-6, IL-1, and TNF- $\alpha$ in the aqueous humour. There was also an elevation in the presence of serum TGF- $\beta_{1}$ in the aqueous humour. Infiltration of cells maximized between 12 and $24 \mathrm{~h}$ after the endotoxin injection. This corresponded with maximum protein concentration in aqueous humour and a loss in the ability of the ocular microenvironment to support ACAID. At $48 \mathrm{~h}$ after endotoxin injection, the ocular microenvironment returned to immunologically normal with normal aqueous humour protein concentration with no detectible proinflammatory cytokines or cells. Also at $48 \mathrm{~h}$, ACAID could be induced, and aqueous humour had its expected immunosuppressive activity. It was concluded that before there is a break in blood-ocular barrier, there is a change in the immunosuppressive properties of the ocular microenvironment immediately following a systemic injection of endotoxin. It is in this leaky and proinflammatory ocular microenvironment where ACAID cannot be supported.

In EAU, some similar changes with EIU were observed. ${ }^{33}$ Before the onset of detecting a retinal infiltration, aqueous humour lost its immunosuppressive properties, and proinflammatory cytokines were again found in the aqueous humour. Interestingly, although the presence of proinflammatory cytokines were found in aqueous humour up to the peak of disease, aqueous humour recovered its immunosuppressive properties well before the maximum retinal inflammation was reached. In addition, the ocular microenvironment stopped supporting ACAID at the onset of retinal inflammation, and did not recover its ability to promote ACAID until after the disease started to resolve. The presence of cells in the aqueous humour followed exactly the course of retinal inflammation. Once the retinal inflammation started to resolve, there was a decline in cells and proinflammatory cytokines in aqueous humour, and ACAID was recovered. These findings suggested that during a directed attack on the ocular tissue that inflammatory response accelerates as aqueous humour ceases to be immunosuppressive; however, this property of aqueous humour in the inflamed eye is short lived, and could be an indication of how the ocular microenvironment tries in uveitis to impose and to recover immune privilege. In parallel to the clinical observations of EAU, there is a sequential induction of detectible T-cell subsets. ${ }^{29,34-36}$ Very early in the disease and as a result of the immunization, there is the induction of autoantigen-specific Th1 cells, and as the disease progresses, they are either joined by or replaced with autoantigen-specific Th17 cells. At some time in the disease, there emerges the presence of autoantigen-specific $\mathrm{T}_{\text {reg }}$ cells. Interestingly, the resolution of EAU is not dependent on the induction of the $\mathrm{T}_{\text {reg }}$ cells.

Such findings add to how we can understand the progress of uveitis, and on where it maybe possible to target immunity to suppress the progression of uveitis and possibly prevent its induction. The most interesting part of this systematic cataloguing of changes in the uveitic ocular microenvironment overtime is the discovery that the ocular microenvironment attempts to reimpose immunosuppression, which is very successful in mice. How this is possible could lead to a better understanding of what mechanisms are important in maintaining immune privilege and the causes of recurring uveitis in humans.

Now that four generations of ocular immunologists have dissected the mechanisms of immune privilege, although not all mechanisms have been discovered, what does ocular immune privilege mean? It still stands that Medawar's original findings that the ocular microenvironment affords prolonged allograft survival is a feature of the ocular immune privilege. The bloodocular barrier is a necessary feature of the tissue microenvironment, and the lack of direct lymphatic drainage has an important role in immune cell migration out of the eye. What we can add to this list of anatomically related and passive mechanisms are the 
mechanisms that actively engage immune cells. These active mechanisms are mostly identifiable proteins expressed on the surface or are secreted by the cells that make up the ocular microenvironment. These proteins modulate a variety of responses in immune cells from inducing apoptosis to promoting anti-inflammatory cytokine production, and by changing the immune response to specific antigens.

Even with the advancements we have made in understanding the molecular mechanisms of ocular immune privilege, there still remain many unanswered questions, the answering of which will lead us to further discoveries and possible further redefinition of what ocular immune privilege means. We still have very little understanding of what mechanism of ocular immune privilege are lost or altered that causes uveitis, and whether it is different depending on the type of immunity that targets the ocular tissues. Also, it is unknown as to how different infectious agents affect ocular immunity. Recently, some initiating attempts have been made to see whether ocular immune privilege can be restored by reintroducing the immunomodulating neuropeptides. ${ }^{37-39}$ One aspect of studying ocular immune privilege is that we have assumed that it is the same throughout the eye. This is based on the findings that antigen placed in the anterior chamber, vitreous, or subretinal space all lead to the induction of an ACAIDlike response. ${ }^{40-42}$ However, this does not show that the regulation of other immune responses in the eye is the same in all regions of the ocular microenvironment. This nanomicroenvironment regulation of immunity is suggested by the difference in pigmented epithelial cells of the retina and iris/ciliary body to suppress T-cell activation through contact and soluble factors. ${ }^{43}$ In addition, there is a differential expression of neuropeptide production between their release by neurons of the anterior chamber and their local production in the retina. ${ }^{44,45}$ Finally, if the mechanisms of ocular immune privilege is mediated by soluble factors and surface proteins, is it possible to impart some of these mechanisms on other tissues to suppress autoimmune disease and graft rejection? Being able to accomplish this translation of the mechanisms of ocular immune privilege will drastically change treatments, and greatly improve the clinical outcomes of autoimmune diseases and allograft survival. ${ }^{46}$

Having a need to minimize the collateral damage of inflammation and infection to preserve the clarity of the visual axis, evolution has adapted several anatomical and biochemical mechanisms to establish an immune privileged microenvironment within the eye. It is this uniqueness of the ocular microenvironment where we can find new and innovative approaches to regulate immunity in inflammation and at rest.

\section{Acknowledgements}

This study was funded by the PHS Grant EY010752.

\section{References}

1 Medawar P. Immunity to homologous grafted skin. III. the fate of skin homografts transplanted to the brain to subcutaneous tissue, and to the anterior chamber of the eye. Br J Exp Pathol 1948; 29: 58-69.

2 Barker CF, Billingham RE. The role of afferent lymphatics in the rejection of skin homografts. J Exp Med 1968; 128: 197-221.

3 Kaplan HJ, Streilein JW. Immune response to immunization via the anterior chamber of the eye. I. $F_{1}$ lymphocyte induced-immune deviation. J Immunol 1977; 118: 809-814.

4 Streilein JW. Ocular immune privilege: the eye takes a dim but practical view of immunity and inflammation. J Leukoc Biol 2003; 74: 179-185.

5 Wilbanks GA, Streilein JW. Distinctive humoral immune responses following anterior chamber and intravenous administration of soluble antigen. Evidence for active suppression of IgG2-secreting B lymphocytes. Immunology 1990; 71: 566-572.

6 Lin HH, Faunce DE, Stacey M, Terajewicz A, Nakamura T, Zhang-Hoover J et al. The macrophage F4/80 receptor is required for the induction of antigen-specific efferent regulatory T cells in peripheral tolerance. J Exp Med 2005; 201: $1615-1625$.

7 Sonoda KH, Stein-Streilein J. Ocular immune privilege and CD1d-reactive natural killer T cells. Cornea 2002; 21 S33-S38.

8 D'Orazio TJ, Niederkorn JY. Splenic B cells are required for tolerogenic antigen presentation in the induction of anterior chamber-associated immune deviation (ACAID). Immunology 1998; 95: 47-55.

9 Kaplan HJ, Streilein JW. Immune response to immunization via the anterior chamber of the eye. I. $F_{1}$ lymphocyte induced-immune deviation. J Immunol 1977; 118: 809-814.

10 Wenkel H, Streilein JW, Young MJ. Systemic immune deviation in the brain that does not depend on the integrity of the blood-brain barrier. J Immunol 2000; 164: 5125-5131.

11 Wilbanks GA, Streilein JW. Fluids from immune privileged sites endow macrophages with the capacity to induce antigen-specific immune deviation via a mechanism involving transforming growth factor-beta. Eur J Immunol 1992; 22: 1031-1036.

12 Kaiser CJ, Ksander BR, Streilein JW. Inhibition of lymphocyte proliferation by aqueous humor. Reg Immunol 1989; 2: 42-49.

13 Taylor AW, Alard P, Yee DG, Streilein JW. Aqueous humor induces transforming growth factor-beta (TGF-beta)producing regulatory T-cells. Curr Eye Res 1997; 16: 900-908.

14 Taylor AW, Streilein JW, Cousins SW. Identification of alpha-melanocyte stimulating hormone as a potential immunosuppressive factor in aqueous humor. Curr Eye Res 1992; 11: 1199-1206.

15 Taylor AW, Streilein JW, Cousins SW. Immunoreactive vasoactive intestinal peptide contributes to the immunosuppressive activity of normal aqueous humor. J Immunol 1994; 153: 1080-1086.

16 Taylor AW, Yee DG, Streilein JW. Suppression of nitric oxide generated by inflammatory macrophages by calcitonin 
gene-related peptide in aqueous humor. Invest Ophthalmol Vis Sci 1998; 39: 1372-1378.

17 Taylor AW, Yee DG. Somatostatin is an immunosuppressive factor in aqueous humor. Invest Ophthalmol Vis Sci 2003; 44: 2644-2649.

18 Ryu YH, Kim JC. Expression of indoleamine 2,3-dioxygenase in human corneal cells as a local immunosuppressive factor. Invest Ophthalmol Vis Sci 2007; 48: 4148-4152

19 Griffith TS, Brunner T, Fletcher SM, Green DR, Ferguson TA Fas ligand-induced apoptosis as a mechanism of immune privilege. Science 1995; 270: 1189-1192.

20 Taylor AW. The immunomodulating neuropeptide alpha-melanocyte stimulating hormone (a-MSH) suppresses LPS-stimulated TLR4 with IRAK-M in macrophages. J Neuroimmunol 2005; 162: 43-50.

21 Catania A, Rajora N, Capsoni F, Minonzio F, Star RA, Lipton JM. The neuropeptide alpha-MSH has specific receptors on neutrophils and reduces chemotaxis in vitro. Peptides 1996; 17: 675-679.

22 Masli S, Turpie B, Hecker KH, Streilein JW. Expression of thrombospondin in TGFbeta-treated APCs and its relevance to their immune deviation-promoting properties. J Immunol 2002; 168: 2264-2273.

23 Apte RS, Sinha D, Mayhew E, Wistow GJ, Niederkorn JY. Cutting edge: role of macrophage migration inhibitory factor in inhibiting NK cell activity and preserving immune privilege. J Immunol 1998; 160: 5693-5696.

24 Jha P, Bora PS, Sohn JH, Kaplan HJ, Bora NS. Complement system and the eye. Adv Exp Med Biol 2006; 586: 53-62.

25 Zamiri P, Masli S, Kitaichi N, Taylor AW, Streilein JW. Thrombospondin plays a vital role in the immune privilege of the eye. Invest Ophthalmol Vis Sci 2005; 46: 908-919.

26 Cousins SW, Trattler WB, Streilein JW. Immune privilege and suppression of immunogenic inflammation in the anterior chamber of the eye. Curr Eye Res 1991; 10: 287-297.

27 Nishida T, Taylor AW. Specific aqueous humor factors induce activation of regulatory T cells. Invest Ophthalmol Vis Sci 1999; 40: 2268-2274.

28 Sugita S, Keino H, Futagami Y, Takase H, Mochizuki M, Stein-Streilein J et al. B7 + iris pigment epithelial cells convert T cells into CTLA-4 +, B7-expressing CD8 + regulatory T cells. Invest Ophthalmol Vis Sci 2006; 47: 5376-5384.

29 Kitaichi N, Namba K, Taylor AW. Inducible immune regulation following autoimmune disease in the immuneprivileged eye. J Leukoc Biol 2005; 77: 496-502.

30 Streilein JW, Masli S, Takeuchi M, Kezuka T. The eye's view of antigen presentation. Hum Immunol 2002; 63: 435-443.

31 Taylor AW, Streilein JW, Cousins SW. Alpha-melanocytestimulating hormone suppresses antigen-stimulated T cell production of gamma-interferon. Neuroimmunomodulation 1994; 1: 188-194.

32 Ohta K, Yamagami S, Taylor AW, Streilein JW. IL-6 antagonizes TGF-beta and abolishes immune privilege in eyes with endotoxin-induced uveitis. Invest Ophthalmol Vis Sci 2000; 41: 2591-2599.

33 Ohta K, Wiggert B, Yamagami S, Taylor AW, Streilein JW. Analysis of immunomodulatory activities of aqueous humor from eyes of mice with experimental autoimmune uveitis. J Immunol 2000; 164: 1185-1192.

34 Luger D, Silver PB, Tang J, Cua D, Chen Z, Iwakura Y et al. Either a Th17 or a Th1 effector response can drive autoimmunity: conditions of disease induction affect dominant effector category. J Exp Med 2008; 205: 799-810.

35 Sun B, Rizzo LV, Sun SH, Chan CC, Wiggert B, Wilder RL et al. Genetic susceptibility to experimental autoimmune uveitis involves more than a predisposition to generate a T helper-1-like or a T helper-2-like response. J Immunol 1997; 159: 1004-1011.

36 Yoshimura T, Sonoda KH, Miyazaki Y, Iwakura Y, Ishibashi $\mathrm{T}$, Yoshimura A et al. Differential roles for IFN-gamma and IL-17 in experimental autoimmune uveoretinitis. Int Immunol 2008; 20: 209-214.

37 Nishida T, Miyata S, Itoh Y, Mizuki N, Ohgami K, Shiratori $\mathrm{K}$ et al. Anti-inflammatory effects of alpha-melanocytestimulating hormone against rat endotoxin-induced uveitis and the time course of inflammatory agents in aqueous humor. Int Immunopharmacol 2004; 4: 1059-1066.

38 Shiratori K, Ohgami K, Ilieva IB, Koyama Y, Yoshida K, Ohno S. Inhibition of endotoxin-induced uveitis and potentiation of cyclooxygenase- 2 protein expression by alpha-melanocyte-stimulating hormone. Invest Ophthalmol Vis Sci 2004; 45: 159-164.

39 Bonfiglio V, Camillieri G, Avitabile T, Leggio GM, Drago F. Effects of the COOH-terminal tripeptide alpha-MSH(11-13) on corneal epithelial wound healing: Role of nitric oxide. Exp Eye Res 2006.

40 Anand V, Duffy B, Yang Z, Dejneka NS, Maguire AM, Bennett $\mathrm{J}$. A deviant immune response to viral proteins and transgene product is generated on subretinal administration of adenovirus and adeno-associated virus. Mol Ther 2002; 5: 125-132.

41 Wenkel H, Chen PW, Ksander BR, Streilein JW. Immune privilege is extended, then withdrawn, from allogeneic tumor cell grafts placed in the subretinal space. Invest Ophthalmol Vis Sci 1999; 40: 3202-3208.

42 Sonoda KH, Sakamoto T, Qiao H, Hisatomi T, Oshima T, Tsutsumi-Miyahara $\mathrm{C}$ et al. The analysis of systemic tolerance elicited by antigen inoculation into the vitreous cavity: vitreous cavity-associated immune deviation. Immunology 2005; 116: 390-399.

43 Ishida K, Panjwani N, Cao Z, Streilein JW. Participation of pigment epithelium in ocular immune privilege. 3. Epithelia cultured from iris, ciliary body, and retina suppress T-cell activation by partially non-overlapping mechanisms. Ocul Immunol Inflamm 2003; 11: 91-105.

44 Larsen JN, Bersani M, Olcese J, Holst JJ, Moller M. Somatostatin and prosomatostatin in the retina of the rat: an immunohistochemical, in-situ hybridization, and chromatographic study. Vis Neurosci 1990; 5: 441-452.

45 Ye XD, Laties AM, Stone RA. Peptidergic innervation of the retinal vasculature and optic nerve head. Invest Ophthalmol Vis Sci 1990; 31: 1731-1737.

46 Streilein JW. Ocular immune privilege: therapeutic opportunities from an experiment of nature. Nat Rev Immunol 2003; 3: 879-889. 